\title{
LETTER
}

\section{Living fast and dying of infection: host life history drives interspecific variation in infection and disease risk}

Pieter T. J. Johnson, ${ }^{1 *}$ Jason R. Rohr, ${ }^{2}$ Jason T. Hoverman, ${ }^{1}$ Esra Kellermanns, ${ }^{1}$ Jay Bowerman ${ }^{3}$ and Kevin B. Lunde ${ }^{4}$

\begin{abstract}
Parasite infections often lead to dramatically different outcomes among host species. Although an emerging body of ecoimmunological research proposes that hosts experience a fundamental trade-off between pathogen defences and life-history activities, this line of inquiry has rarely been extended to the most essential outcomes of host-pathogen interactions: namely, infection and disease pathology. Using a comparative experimental approach involving 13 amphibian host species and a virulent parasite, we test the hypothesis that 'pace-of-life' predicts parasite infection and host pathology. Trematode exposure increased mortality and malformations in nine host species. After accounting for evolutionary history, species that developed quickly and metamorphosed smaller ('fast-species') were particularly prone to infection and pathology. This pattern likely resulted from both weaker host defences and greater adaptation by parasites to infect common hosts. Broader integration between life history theory and disease ecology can aid in identifying both reservoir hosts and species at risk of disease-driven declines.
\end{abstract}

\section{Keywords}

Amphibian decline, conservation, dilution effect, emerging disease, immunoecology, phylogenetic comparative methods, Ribeiroia ondatrae, trematode.

Ecology Letters (2012) 15: 235-242

\section{INTRODUCTION}

Many infectious diseases exhibit pronounced variation in the types and severity of pathology among infected host species (Ostfeld \& Keesing 2000; Kilpatrick et al. 2006; Craft et al. 2008; Cronin et al. 2010). Even co-occurring host species in the same community can exhibit remarkable differences in infection and disease-related symptoms. For instance, following the introduction of West Nile virus into the USA in 1999, mortality among infected bird species varied from near complete (e.g. American crows and other corvids) to non-detectable (LaDeau et al. 2007). Because disease represents the product of ecological and evolutionary interactions between host and parasite, identifying the relative importance of factors contributing to interspecific variation in pathology represents a formidable challenge. Species may differ in infection owing to differences in parasite exposure during their lifetime, which can be influenced by habitat use, geographical distribution, phenology, body size, diet, network structure and behaviour (Lee 2006; Borer et al. 2009; Cronin et al. 2010). In addition, hosts can exhibit differential defences against infection, including variation in immunity, behaviour, stress and physiology (Hart 1994; Schmid-Hempel \& Ebert 2003; Hasselquist 2007). However, identifying the relative importance of parasite exposure vs. host defences may not be straightforward; in many cases, disease variation is likely due to differences in both factors.

Recently, growing interest has focused on the utility of the 'pace-oflife' continuum for explaining interspecific variation in host-parasite interactions (Lochmiller \& Deerenberg 2000; Martin et al. 2006b). In ecoimmunology, for instance, an emerging paradigm centres on lifehistory tradeoffs with investment in pathogen defences coming at the expense of other physiological activities, such as growth and reproduction (Stearns 1992; Ricklefs \& Wikelski 2002). 'Fast-lived' species with rapid growth and short life spans are hypothesised to invest relatively little in defences in favour of growth and early reproduction (Martin et al. 2006b; Lee et al. 2008), whereas 'slow-lived' species, whose longer life spans and slower growth increase pathogen exposure, invest more resources into costly defences (Schmid-Hempel \& Ebert 2003; Martin et al. 2006b; Lee et al. 2008). Concurrently, disease biologists working on fishes, mammals, plants and even humans have increasingly reported linkages between host life history traits and patterns of infection in nature, with slow-lived species often supporting a higher diversity, prevalence, or intensity of infections (e.g. Poulin \& Morand 2004; Arriero \& Moller 2008; Blackwell et al. 2010).

Thus far, however, surprisingly few studies have extended this traitbased approach to examine the role of host life history in determining infection success and its primary fitness outcomes: namely, pathology and disease (Viney et al. 2005; Martin et al. 2007; Hawley \& Altizer 2010). Given the broad diversity of specific and non-specific host defences, a recurring critique focuses on the validity of linking immunological assays with ecologically relevant patterns of disease (see Schmid-Hempel \& Ebert 2003; Viney et al. 2005; Martin et al. 2006b, 2007; Rohr et al. 2010) and may be one reason why different assays can produce equivocal or contradictory results (Tieleman et al.
${ }^{1}$ Ecology and Evolutionary Biology, University of Colorado, Boulder, CO 80309, USA

${ }^{2}$ Department of Integrative Biology, University of South Florida, 4202 East Fowler Ave., Tampa, FL 33620, USA
${ }^{3}$ Sunriver Nature Center, Box 3533, Sunriver, OR 97707, USA

${ }^{4}$ Environmental Science, Policy, and Management, University of California at Berkeley, Berkeley, CA 94720, USA

*Correspondence: E-mail: pieter.johnson@colorado.edu 
2005; Martin et al. 2006a). Similarly, correlational field studies of infection often obscure whether associations with particularly lifehistory characteristics are due to differences in exposure, host defences, or both (Poulin \& Morand 2004). These observations underscore the value of experimental research that can effectively decouple the roles of parasite exposure and host defences over ecological time scales (Komar et al. 2003; Martin et al. 2007). Ideally, such studies should include a diverse assemblage of host species with widely varying life-history traits while controlling for their shared evolutionary history, which can strongly influence infection outcomes (Gilbert \& Webb 2007). For instance, in a noteworthy exception to this general research gap, Cronin et al. (2010) used a phylogenetic approach with six plant species to experimentally test the hypothesis that host physiological phenotype best explained reservoir potential, including hosts' susceptibility to infection and their capacity to support infected vector populations. How such patterns extend to host pathology, particularly for animal disease systems, remains conjectural. Thus, an important yet underexplored complementary line of inquiry involves examining the role of life history in predicting disease pathology, which represents the integrated product of parasite exposure and host defences over time and across space.

Here, we used a comparative experimental approach to test the hypothesis that host pace-of-life can be used to explain epidemiological variation in infection and pathology among a diverse assemblage of host species. We focus on amphibian hosts, whose broad variation in both life-history traits and disease patterns (Todd 2007) make them a valuable system in which to investigate the nexus between these topics. Using a standardised infection protocol, we exposed 13 species of frogs, toads, and salamanders to multiple dosages of the virulent trematode, Ribeiroia ondatrae. Building from life history theory and ecoimmunological research, we experimentally tested the hypothesis that fast-lived species with rapid growth would exhibit greater pathology and be more susceptible hosts relative to slow-lived species with greater investment in somatic maintenance (Lee 2006; Martin et al. 2006b). Given the role of multi-host pathogens in emerging infections of both plants and animals, understanding the drivers of disease variation has immediate relevance for human health, economic growth and wildlife conservation (Daszak et al. 2000; LoGiudice et al. 2003; Jones et al. 2008; Cronin et al. 2010).

\section{MATERIALS AND METHODS}

\section{Study system}

Ribeiroia ondatrae (hereafter 'Ribeiroia') is a pathogenic trematode that causes developmental malformations such as extra, missing or misshapen limbs (Johnson et al. 1999; Rohr et al. 2010). The complex life cycle of Ribeiroia involves freshwater snails, amphibians and water birds. Following their release from snails, free-living parasite cercariae can be counted and administered in specific dosages to amphibians, which facilitates experimental study of this system. Unlike microparasitic infections, larval trematodes do not reproduce within amphibian intermediate hosts, such that infection success and pathology can be quantified independently.

\section{Animal collection and husbandry}

We obtained egg masses or early stage larvae of 11 anuran and two caudate species representing six families (see Table S1). With the exception of $X$. laevis, which was included as an out-group, all species are native to North America and likely overlap with Ribeiroia in nature; individuals of all but one species have been reported with infection or malformations, indicating these are ecologically relevant host-parasite pairings (Table S2). We focused on lentic-breeding amphibians because they encounter trematodes, such as Ribeiroia, during larval development. After hatching, amphibian larvae were raised in the laboratory until they reached early limb development (see Supporting Information).

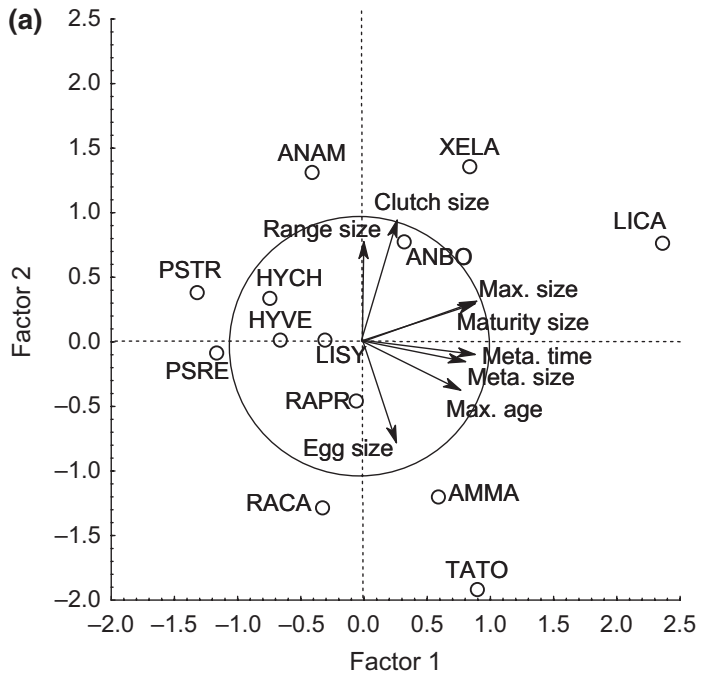

(b)

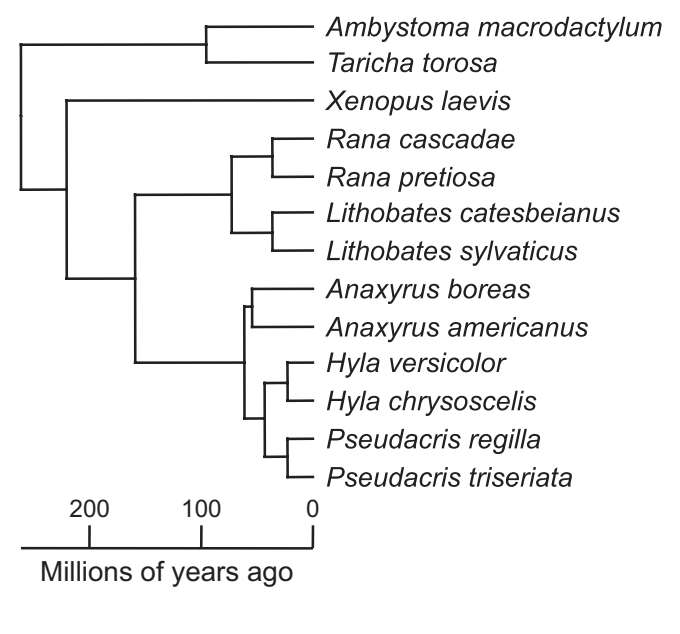

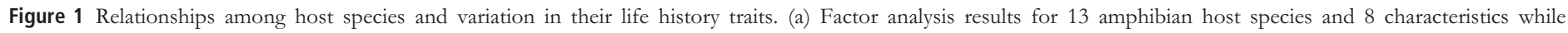

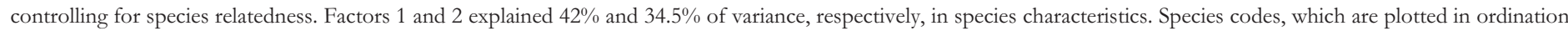

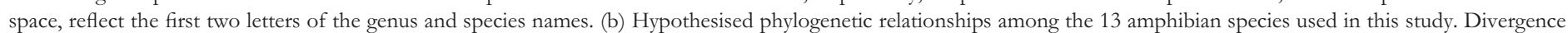

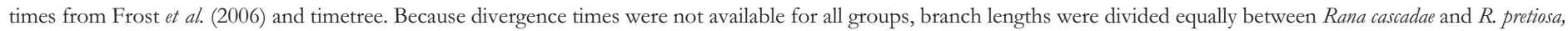
Lithobates catesbeianus and L. sylvaticus, Hyla versicolor and H. chrysoscelis, and Pseudacris regilla and P. triseriata. 


\section{Parasite exposures}

Ribeiroia cercariae were harvested from infected snails (Helisoma trivolvis) within $5 \mathrm{~h}$ of release $(\sim 2100-0200 \mathrm{~h})$ and administered to larval amphibians four times over a 10-day period (days 1, 4, 7 and 10) to better reflect the continuous exposures experienced in nature. Larvae were exposed individually to parasites in a small volume of water, such that all cercariae were generally successful in penetrating the hosts (see Supporting Information). Each amphibian species was exposed to between four and six doses of cercariae, ranging from 0 (control) to 200 or more ( $n \sim 25$ amphibians per treatment) ( $n=1885$ total individuals, Table S1). Because amphibian host species varied in sensitivity to infection, we adjusted parasite exposure dosage among species to ensure an adequate range in pathology responses (e.g. Fig. 2). Following parasite exposures, amphibians were raised to metamorphosis, measured (snout-vent length, SVL), massed (g), and examined for morphological abnormalities (following Johnson et al. 2001). A subset of metamorphic animals in each treatment and animals that died prior to metamorphosis were necropsied to quantify the number and position of encysted Ribeiroia metacercariae within hosts (Johnson \& Hartson 2009). Since experiments were conducted over a 13-year period (1998-2010), there was some variation in experimental protocol among species, which is discussed in detail in the Supporting Information alongside additional details on parasite sources, exposure methods and animal husbandry.

\section{Within-species analyses}

We evaluated the effects of parasite exposure on host survival, malformation frequency, time-to-metamorphosis, length- and mass-atmetamorphosis and parasite load at metamorphosis. We used Firthcorrected logistic regression with a binomially distributed error and a logit-link function to quantify the influence of cercarial dose on survival and malformations. Among malformed animals, we assessed the effects of exposure on abnormality severity, or the number of abnormalities per abnormal animal (Johnson et al. 2001), and the (a) Lithobates sylvaticus

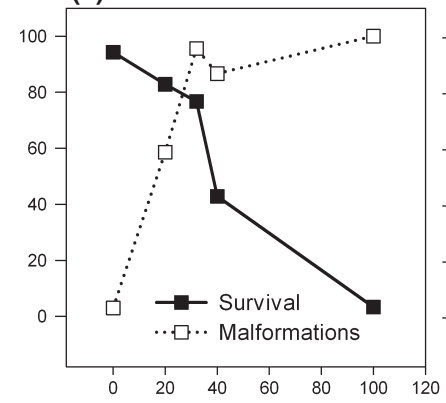

(e) Pseudacris regilla

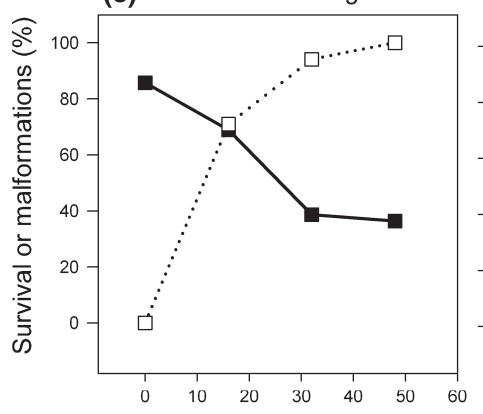

(i) Anaxyrus americanus

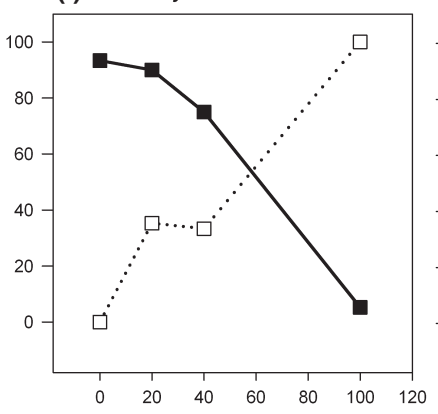

(b)

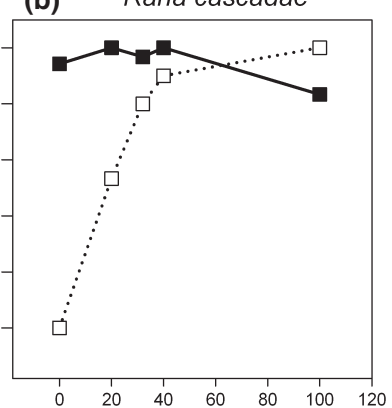

(f) Pseudacris triseriata

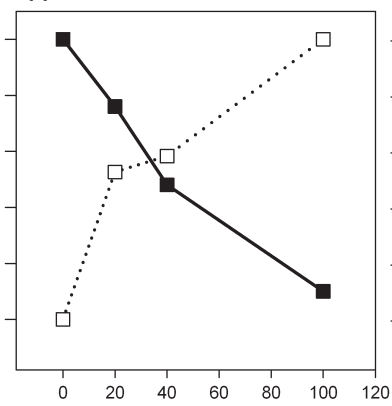

(j) Anaxyrus boreas

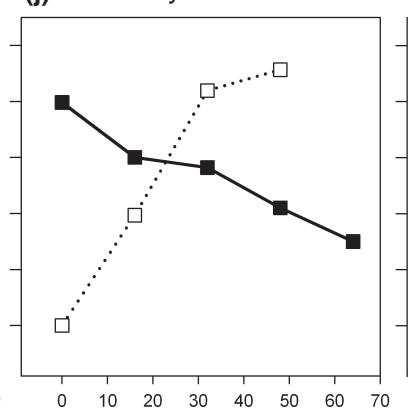

(c)

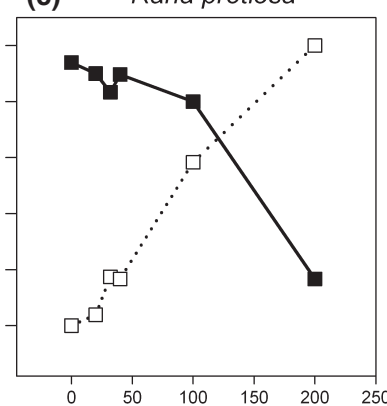

(g) Hyla versicolor

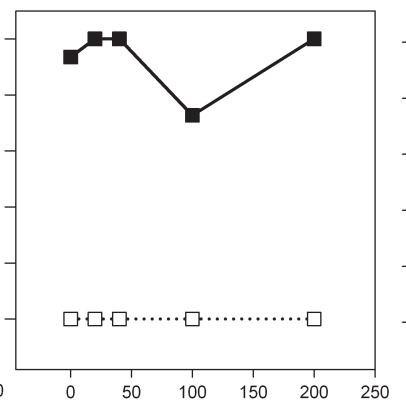

(k) Taricha torosa

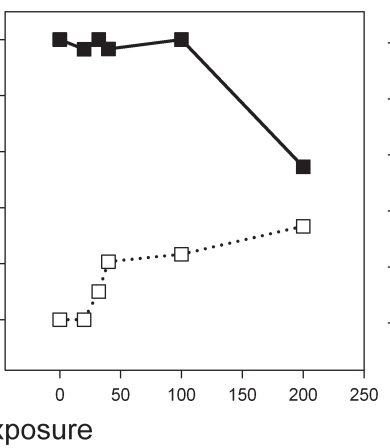

(d)

d) Xenopus laevis

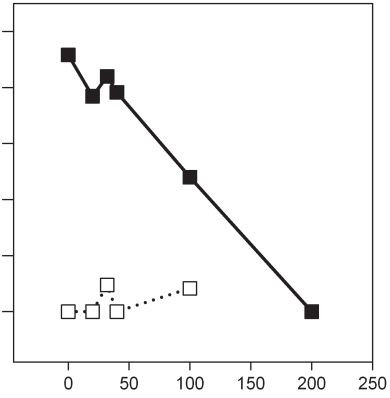

(h) Hyla chrysoscelis

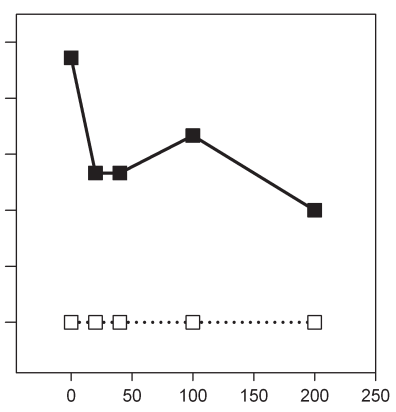

(I) Ambystoma macrodactylum

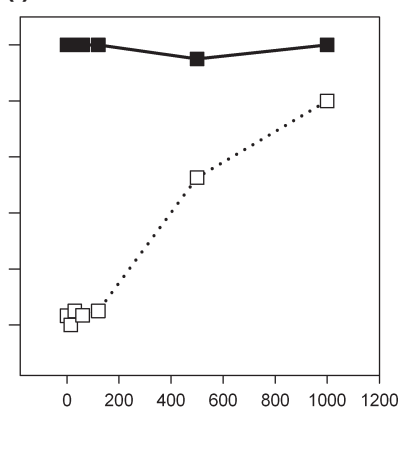

Figure 2 Effects of Ribeiroia ondatrae exposure on the survival and malformation frequency of amphibian hosts. Survival represents the percentage of individuals alive at metamorphosis although malformations were assessed only among survivors. Ribeiroia exposure ( $x$-axis) reflects the number of trematode cercariae to which hosts were exposed and varied to incorporate differences in sensitivity among species. Data for Lithobates catesbeianus are not shown. 
similarity of malformation types among species and treatments (see Supporting Information). Because host length and mass are strongly influenced by time-to-metamorphosis, we evaluated these responses both with and without days-to-metamorphosis as a covariate using ANCOVA. To assess the influence of exposure dosage and host species on patterns of infection, we analysed parasite load at metamorphosis as both a raw value (number of metacercariae, $\log _{10}$-transformed) and as a proportion (number detected/number administered, arcsin-square root transformed). This analysis assumes that most cercariae are successful in penetrating the hosts, which is supported by observation and previous research (see Supporting Information).

\section{Between-species analyses}

We assessed the role of host life history traits in explaining three major response variables: proportion of administered parasites detected at metamorphosis (arcsine-square root transformed), mortality risk (ln-transformed values of the mortality odds ratio) and malformation risk (ln-transformed values of the malformation odds ratio). We used the odds ratio rather than measures such as the LC50 because, when dealing with null effects, the odds ratio approaches unity whereas the LC50 approaches infinity. For each species, we collected data on geographical range size $\left(\mathrm{km}^{2}\right)$ and life history characteristics, including maximum body size $(\mathrm{mm})$ size-at-maturity $(\mathrm{mm})$, maximum clutch size, maximum age (years), and average egg size ( $\mathrm{mm}$ ), and phylogenetic relationships from multiple sources (see Supporting Information). We used data from our own control animals (i.e. those not exposed to Ribeiroia) to obtain standardised information on timeto-metamorphosis and length-at-metamorphosis under laboratory conditions. Given that life-history traits and species characteristics are often correlated, we conducted a factor analysis with a varimax rotation (McGarigal et al. 2000) to extract the primary life-history axes (all variables were $\log _{10}$-transformed) after correcting for phylogeny. Species range size was included in the factor analysis because of its relationship to species dispersal and abundance.

Because closely related species might not be truly independent in their responses (Martins \& Hansen 1997), we conducted our analyses using phylogenetic independent contrasts for each response using the 'pic' function in the 'ape' package of $\mathrm{R}$ statistical software (R Development Core Team 2008; Paradis et al. 2011) and then conducted regression analyses on these phylogenetically controlled responses and predictors (excluding the intercept). Branch lengths (i.e. divergence times) for the phylogeny were obtained using TimeTree or published studies (Frost et al. 2006) (Fig. 1b). When divergence times were not available for a group, we divided the branch length equally among the taxa (see Fig. 1 for details). Phylogenetic independent contrasts are a conservative approach to testing for associations between responses and predictors because they consume a degree of freedom (see Supporting Information for additional evaluations of phylogenetic influences, all of which supported the conclusion that the focal response variables did not have a phylogenetic signal; Table S3).

To evaluate whether life-history traits could predict parasite load and pathology among species, we conducted a multiple regression analysis on each of these variables with the first two factors from the factor analysis as predictors. We used path analysis to further test whether each factor influenced pathology (mortality and malformations) directly and/or indirectly through their effects on infection. Path analyses were conducted using the 'sem' function in the 'lavaan' package of R statistical software (R Development Core Team 2008; Rosseel et al. 2011). Finally, to explore which combinations of the eight life-history variables were the best predictors of each of the three response variables, we used an information theoretical approach to evaluate the support of 23 candidate models, including a null model (intercept only), a global model, eight single variable models and combinations of models focused on geographic distribution (range size), larval characteristics (time-to- and size-at-metamorphosis), adult characteristics (maximum age and sizes) and reproductive traits (egg and clutch sizes) (see Table S9). After controlling for phylogeny, we assessed the support for each model based on $\mathrm{AIC}_{\mathrm{c}}$ values and calculated the relative importance of each variable based on cumulative Akaike weights (Burnham \& Anderson 2002).

\section{RESULTS}

\section{Survival and malformations}

As predicted, Ribeiroia exposure increased the likelihood of host mortality and malformations. Mortality, which varied from 0 to $100 \%$ among treatments, was influenced by parasite dosage, host species identity and their interaction $\left(\chi^{2}=636.39, P<0.0001, n=1885\right.$; Fig. 2). Exposure to Ribeiroia increased mortality in 9 of the 13 amphibian species in a dose-dependent manner (Fig. 2, Table S4). Mortality was highest for the toads, chorus frogs and small-bodied ranids (Fig. 2), whereas Ribeiroia had no effect on the survival of A. macrodactylum, Hyla chrysoscelis, H. versicolor, or Lithobates catesbeianus (all $P \geq 0.059$ ). Among animals that metamorphosed, parasite exposure increased the risk of malformations for 9 of the 13 species $\left(\chi^{2}=862.04\right.$, d.f. $=25, P<0.0001, n=1384$; Fig. 2$)$, including eight of the nine species that exhibited a survival effect (see Table S5). Malformations were most common among toads, chorus frogs and small-bodied ranids (Fig. 2). For species with $>20$ abnormal animals (six species), increases in parasite exposure also correlated positively with abnormality severity (see Supporting Information). Parasiteinduced malformations were dominated by cutaneous fusion $(22.9 \%)$, extra limbs $(18.9 \%)$ and digits $(7.8 \%)$, bony triangles $(18.0 \%)$, missing limbs, feet or digits $(7.8 \%)$ and micromelia $(5.9 \%)$, although these patterns varied considerably among species (Table S6). Few or no abnormalities were observed in $\mathrm{H}$. chrysoscelis, $\mathrm{H}$. versicolor, L. catesbeianus, and Xenopus laevis, and amphibians in the control treatments exhibited high survival (average $=93.9 \pm 1.7 \%$ ) and few malformations (average $=0.5 \pm 0.3 \%$, Supporting Information) (Fig. 2).

\section{Size, mass, and time-to-metamorphosis}

Ribeiroia exposure generally reduced growth and delayed time-tometamorphosis. For $R$. cascadae, $X$. laevis, $A$. americanus, and A. macrodactylum, the standardised coefficient of the per-parasite effect on days-to-metamorphosis ( $\log _{10}$-transformed) ranged from 0.29 to 0.54 (all significant, Table S7). After accounting for the effects of parasitism on time-to-metamorphosis, Ribeiroia also reduced amphibian length-at-metamorphosis for $R$. cascadae, $T$. torosa, and $X$. laevis and mass-at-metamorphosis for $A$. americanus, $T$. torosa and $X$. laevis (Table S7). $R$. pretiosa exhibited an increase in mass with parasite exposure $\left(F_{2,123}=7.952, \quad P=0.001\right)$. The remaining species showed no significant changes in growth as a function of parasite exposure. 


\section{Parasite load}

Among hosts examined at metamorphosis $(n=423)$, the percentage of administered parasites detected upon necropsy averaged ( $\pm 1 \mathrm{SE}$ ) $25.39 \pm 0.88 \%$ (Table S7). This fraction varied strongly by host species but not by exposure dose (ANOVA $F_{13,409}=40.242$, $P<0.0001$; species [fixed factor]: $F_{12,409}=43.57, \quad P<0.0001$; dosage [covariate]: $\left.F_{1,409}=0.721, P=0.396\right)$, with the lowest infections in the two Hyla species $(<1 \%)$ and the highest parasite load in $P$. regilla and the small-bodied ranids (29-41.6\%) (Table S8). Among animals for which we had data on infection and time-tometamorphosis $(n=333)$, the number of parasites varied by species $\left(F_{8,298}=28.795, \quad P<0.0001\right), \quad$ exposure level $\left(F_{5,298}=2.726\right.$, $P=0.02)$ and days post-exposure $\left(F_{1,298}=36.381, P<0.0001\right)$; longer development times tended to reduce parasite load, possibly reflecting greater time for host clearance. Even among species that exhibited low parasite loads $(<1 \%$ of parasites detected at metamorphosis) (e.g. H. chrysoscelis), $\sim 18.7 \%$ of exposed parasites were detected as metacercariae in tadpoles that died within $72 \mathrm{~h}$ of exposure. This indicates that, although parasites were successful in entering and encysting in the host (see Supporting Information), they were subsequently lost through host immunity or parasite mortality. We note, however, that our study was not designed to differentiate between the relative importance of host immunity and parasite mortality in influencing clearance rate.

\section{Between-species analyses}

The factor analysis yielded two axes that collectively accounted for $77 \%$ of the variance in species traits and generally divided larval development characteristics (Factor 1) from those associated with adult size and reproduction (Factor 2). Time-to-metamorphosis (loading $=0.91$ ), length-at-metamorphosis (loading $=0.83$ ) and maximum age (loading $=0.76)$ each loaded heavily on Factor 1 , which explained $42 \%$ of the variance (Fig. 1A). Range size (loading $=0.81$ ), clutch size (loading $=0.81)$, maximum body size (loading $=0.72$ ) and size-at-maturity (loading $=0.82$ ) loaded on Factor 2 (35\% of the variance). Egg size did not load strongly on either axis. Thus, after controlling for phylogeny (Fig. 1B), species with higher Factor 1 scores were those that developed more slowly and had longer life spans, whereas those with high Factor 2 scores had larger clutch sizes, geographic ranges and maximum body sizes. Based on the regression analyses, species with higher Factor 1 scores had lower parasite loads $\left(F_{1,10}=6.83, P=0.026\right.$; Fig. $\left.3 \mathrm{~A}\right)$, mortality $\left(F_{1,10}=7.25, P=0.023\right.$; Fig. $\left.3 \mathrm{~b}\right)$, and marginally lower malformation $\operatorname{risk}\left(F_{1,10}=4.14, P=0.069\right.$; Fig. $\left.3 \mathrm{c}\right)$. Because the two Hyla species were outliers with no malformations and almost no infection, we repeated the analysis without these species. Despite the decrease in sample size, the results were similar or stronger, suggesting the observed patterns were robust (parasite load: $F_{1,8}=15.70, P=0.004$; mortality risk: $F_{1,8}=7.82$, $P=0.023$; malformation risk: $\left.F_{1,8}=5.21, P=0.052\right)$. Factor 2 did not predict any response variable, regardless of whether Hyla were included (all $P>0.104$ ). Results were also not sensitive to whether we included the out-group, $X$. laevis.

The information theoretic analysis revealed that traits associated with host larval period, such as time-to- and especially length-atmetamorphosis, had the greatest relative importance and lowest $\mathrm{AIC}_{\mathrm{c}}$ values across all response variables, regardless of whether we excluded Hyla (Fig. 4; Tables S10-12). As predicted, both variables correlated negatively with the three epidemiological responses (Table S13), indicating that species with longer larval periods and larger metamorphic sizes had fewer parasites and less pathology. Based on the path analysis, species with higher values on Factor 1 (longer larval periods and larger sizes at metamorphosis) had fewer parasites at metamorphosis $(\beta=-0.623, P=0.006)$ whereas both Factor 1 and parasite load affected mortality risk (Factor $1: \beta=-0.475, P=0.012$; Parasite load: $\beta=0.480, P=0.011)$, indicating that host life history influenced parasite-induced mortality both directly and indirectly. In contrast, Factor 1 affected malformation risk only through its effect on parasite load, which had a strongly positive influence on malformation odds $(\beta=0.898, P<0.001)$.

\section{DISCUSSION}

Results of our comparative experimental approach highlight the importance of host life-history traits in determining parasite infection

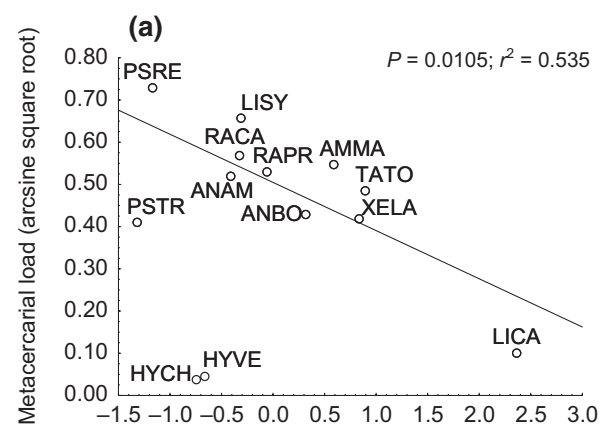

(b)

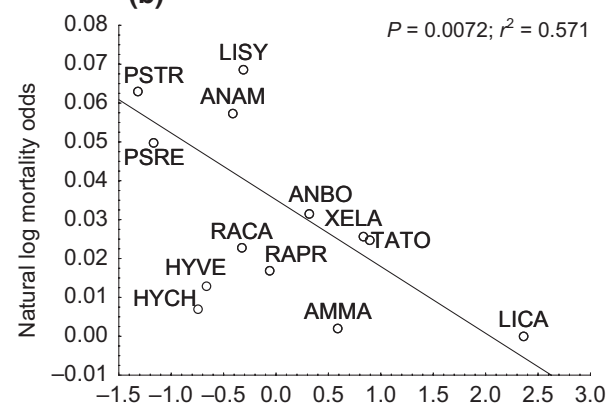

(c)

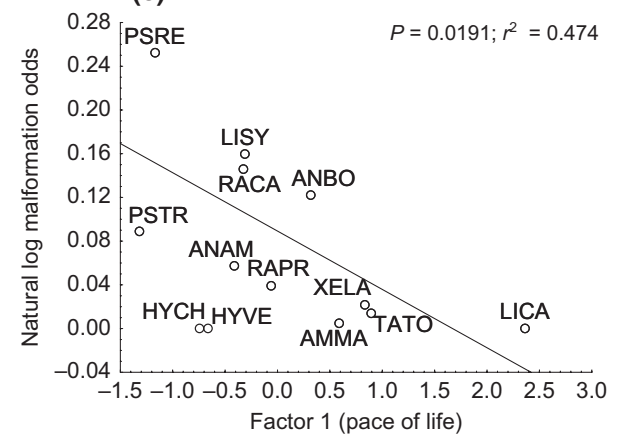

Figure 3 Relationships between Factor 1 from the factor analysis ('pace-of-life' axis) and responses variables: (a) parasite load at metamorphosis (arcsine-square root transformed); (b) mortality risk (ln-transformed odds ratio); (c) malformation risk (ln-transformed odds ratio). Trendlines and associated statistics plotted for each panel with $H$. versicolor and $H$. chyrsoscelis excluded. For ease of presentation, graphs reflect data without correcting for phylogenetic relationships (see text for details). 
success and the resulting patterns of disease pathology. Among 13 amphibian host species, experimental exposures to the virulent trematode Ribeiroia ondatrae revealed dramatic variation in parasite load, host survival and the frequency of malformations. These responses were best predicted by a single life-history axis related to larval growth rate, metamorphic body size and life span. Supporting the hypothesis that host pace-of-life would influence disease responses, species that developed quickly, had smaller sizes at metamorphosis and lived for shorter periods ('fast-lived' species), exhibited the highest levels of both parasite load and pathology. Slowlived species, in contrast, with more gradual growth and larger body sizes, had fewer parasites at metamorphosis and had less severe pathology. Importantly, these results were not an artefact of other factors often hypothesised to explain disease patterns, such as geographical provenance or shared evolutionary history (Gilbert \& Webb 2007; Cronin et al. 2010). Although pace-of-life has been applied previously to understand host investments in immune defences, reservoir potential and conservation status (Purvis et al. 2000), this study is among the first to use a trait-based approach to experimentally explain patterns of infection and disease pathology in an animal system (but see Cronin et al. 2010 for an example from plants).

Our findings complement and build upon an emerging body of research focused at the intersection between life history theory and disease ecology. A fundamental premise of life-history theory and ecoimmunology is that defences are costly and come at the expense of other physiological activities, such as growth and reproduction ('cost of immunity' hypothesis) (Lochmiller \& Deerenberg 2000; Lee 2006; Martin et al. 2006a; Hasselquist 2007). Given that the likelihood of exposure to parasites, as well as the virulence of those parasites, varies both spatially and temporally, hosts ought to experience selective

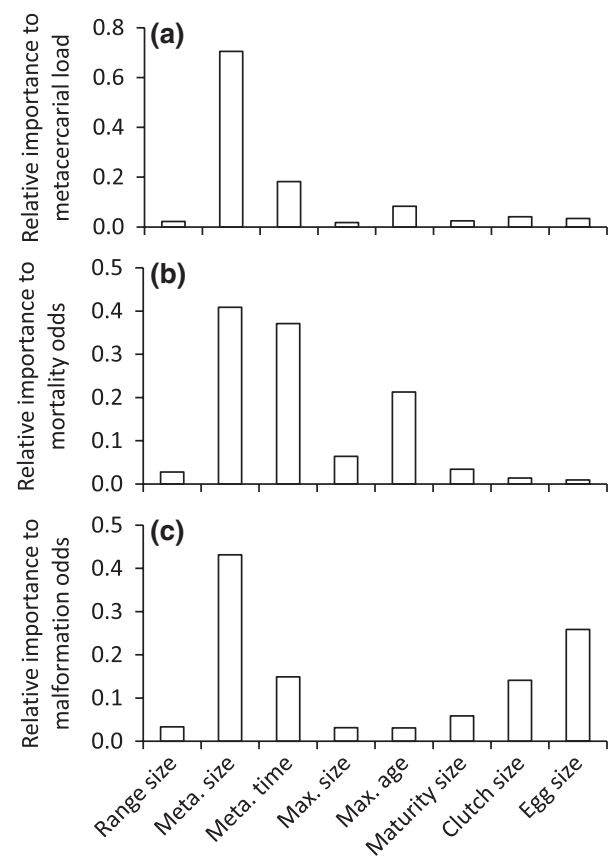

Figure 4 Relative importance of specific life-history traits in explaining host responses to parasite exposure. Cumulative Akaike weights of life-history variables after controlling for phylogeny are presented for (a) parasite load, (b) mortality risk and (c) and malformation risk. pressure to optimise energetic investments into defences as a function of disease risk (Zuk \& Stoehr 2002; Martin et al. 2007). Host species that develop quickly (e.g. toads), or early in the season when infection risk is low (e.g. wood frogs), should invest comparatively little in defences relative to slow-developing species (e.g. bullfrogs and newts) with a much higher likelihood of parasite encounter. Correspondingly, host traits such as body size, development time, clutch size, lifespan and morphology have been found to correlate with host parasitemia or immunological defences in birds, mammals, humans, plants and reptiles (Poulin \& Morand 2004; Martin et al. 2006b, 2007; Lee et al. 2008; Borer et al. 2009; Knowles et al. 2009; Sparkman \& Palacios 2009; Blackwell et al. 2010; Cronin et al. 2010).

In contrast to previous pace-of-life work focused on animal immunology, however, characteristics related to adult reproduction explained relatively little variation in pathology in this study compared to traits associated with larval growth and development (Martin et al. 2006b, 2007; Lee et al. 2008). This suggests that, for larval amphibians exposed to water-borne trematodes, early growth characteristics such as time-to-metamorphosis and size-at-metamorphosis are more salient in determining host defences than those associated with postmetamorphic life, such as size-at-maturity, clutch size, or maximum size. Thus, species that developed quickly as larvae and metamorphosed at small sizes, such as toads, were highly sensitive to parasite exposure, even though they are long-lived and large-bodied as adults. The explanation for this trend is likely twofold. First, small-bodied amphibians that metamorphose quickly might exhibit more infectionrelated damage for the simple reason that the proportional tissue area disrupted by each invading parasite is greater than in large-bodied hosts. Correspondingly, amphibian larvae exposed to Ribeiroia at earlier stages of development (and smaller body sizes) exhibit lower tolerance than late-stage larvae, with greater mortality and malformations (Rohr et al. 2010; Johnson et al. 2011).

Second, host species with a more prolonged larval period have more time to repair infection-related damage, potentially leading to lower parasite persistence and fewer fitness costs at metamorphosis. Because slow-growing, large-bodied species are likely to accumulate more infections during development, including those of virulent pathogens (Poulin \& Morand 2004), life-history theory suggests they ought to invest greater resources into defences relative to fast-paced species that may be able to avoid heavy infection loads (Lee 2006; Martin et al. 2007; Miller et al. 2007). In support of this trade-off hypothesis, a path analysis indicated that host pace-of-life influenced pathology both directly by reducing mortality risk and indirectly by lowering parasite load. Hosts with longer development periods and larger sizes were better able to tolerate infection and, owing to fewer parasites at metamorphosis, limit infection-induced pathology such as mortality. Indeed, pathogen infection risk has been hypothesised as a contributing factor in the evolution of amphibian life history and reproductive strategies, including the progressive evolution of more terrestrial life histories in amphibians to perhaps limit water-borne infections by virulent pathogens (Todd 2007).

Although most work has assumed a 'host-centric' position in evaluating the linkages between life history and disease, emphasising the physiological trade-offs between growth and defences, infection outcomes represent the result of dynamic adaptations by both host and parasite (Råberg et al. 2009), and parasites are unlikely to be static participants in these interactions. A priori, one might expect parasites to progressively specialise on host species that are widely distributed and locally abundant (i.e. r-type species with strong dispersal abilities; 
Ostfeld \& Keesing 2000; Keesing et al. 2006). Given that in many systems these hosts will also be 'fast-paced' species with strong colonisation abilities but weaker defences (Promislow \& Harvey 1990; Stearns 1992), parasite adaptation could function to reinforce the patterns of infection success reported herein. Parasite life history and transmission mode are also likely to influence the types and severity of pathology observed in hosts. In the Ribeiroia system, malformations are predicted to enhance transmission by increasing the likelihood that infected amphibians are consumed by definitive host predators (Johnson et al. 1999); thus, host species with high parasite loads and severe malformations will be most effective at completing the parasite life cycle. For other modes of transmission dependent on host contact rates or vector exposure, however, pathology may be less important than host competency and infectivity when dealing with infections that involve reservoir hosts (LoGiudice et al. 2003; Martin et al. 2007; Cronin et al. 2010). This suggests that the relationship between host pace-of-life and disease pathology will be critically mediated by parasite traits.

Consistent with previous research on the effects of Ribeiroia on amphibian hosts, exposure to increasing doses of cercariae caused a wide range of pathologies, including reduced survival (nine of 13 species), increased limb malformations (nine species), and reduced growth rates or size-at-metamorphosis (six species) (Johnson et al. 1999, 2001, 2011). Although host pace-of-life and exposure dosage explained much of the variation in infection and pathology, these relationships were decoupled in certain species. For instance, few malformations were observed in $X$. laevis or $T$. torosa, despite reasonably high infection loads and mortality, whereas $R$. cascadae exhibited a high malformation frequency and parasite load but little mortality. Most notably, the grey tree frog species complex (Hyla versicolor and $H$. chrysoscelis) emerged as outliers to the general relationship between life history and disease, with $<1 \%$ of parasites recovered at metamorphosis and low frequencies of mortality and malformations, despite intermediate development times and body sizes. Even the out-group $X$. laevis, which is endemic to Africa and has no evolutionary history with $R$. ondatrae, exhibited more infection and mortality than did grey tree frogs. This suggests that, while life-history traits correlate broadly with host responses to parasite exposure, there are likely multiple, alternative defence strategies involving concurrent variation in both resistance and tolerance, as supported by recent theoretical studies (Miller et al. 2007).

Continued research at the interface between life-history theory and disease ecology has the potential to help integrate diverse topics of both conceptual and applied importance, including the relationship between biodiversity and disease risk, identification of reservoir hosts and disease-driven declines in threatened hosts (Ostfeld \& Keesing 2000; LoGiudice et al. 2003; Kilpatrick et al. 2006; Cronin et al. 2010). Reservoir hosts, which are classified according to their susceptibility to infection and ability to maintain infections, have also been linked to pace-of-life and defence investments (Haydon et al. 2002; Komar et al. 2003; LoGiudice et al. 2003; Borer et al. 2009; Cronin et al. 2010). For instance, LoGuidice et al. (2003) found that small-bodied and shortlived vertebrates, such as mice, shrews and chipmunks, functioned as the most competent reservoirs for Lyme disease, efficiently maintaining the infection and transmitting it to tick vectors. These species also tended to predominate in low diversity communities (Ostfeld \& Keesing 2000). In one of the few comparative life-history studies to experimentally infect hosts, Cronin et al. (2010) showed that plants with 'quick return' phenotypes with higher nutrient concentrations and fewer leaf defences were most susceptible to a vector-borne virus, suggesting the exciting potential for cross-kingdom comparisons of interactions among life history, infection and disease. Given that this effort will require broader comparisons of the linkages between paceof-life and disease for a wide range of both host and pathogen taxa, a greater emphasis on experimental research that accounts for exposure and host defences is warranted. Taken together, these observations underscore the importance of continued research integrating disease ecology, host immunology and life history theory, not only for understanding the interactions between hosts and parasites but also for managing disease and promoting conservation (Ricklefs \& Wikelski 2002; Hawley \& Altizer 2010).

\section{ACKNOWLEDGEMENTS}

For assistance in conducting experiments and collecting data, we thank E. Daly, S. Todd, R. Hartson, E. Preu, M. Redmond, M. McGrath, G. Cropsey, E. Holldorf, C. de Jesus, J. Jenkins, I. Buller, D. Larson, A. Price and B. LaFonte. Comments from R. Ostfeld, J. Cronin, two anonymous referees and members of the Johnson Lab helped shape the manuscript. M. Lajeunesse generously provided help with the phylogenetic analyses. This project was supported by grants from NSF (DEB-0553768 to PTJJ), the US Department of Agriculture (NRI 2006-01370, 2009-35102-05043 to JRR), the US Environmental Protection Agency (R833835 to JRR) and a fellowship from the David and Lucile Packard Foundation (to PTJJ).

\section{AUTHORSHIP}

PTJJ designed research, PTJJ, EK, JB and KBL performed research, PTJJ, JRR and JTH analysed data, and all authors wrote the manuscript.

\section{REFERENCES}

Arriero, E. \& Moller, A.P. (2008). Host ecology and life-history traits associated with blood parasite species richness in birds. J. Evol. Biol., 21, 1504-1513.

Blackwell, A.D., Snodgrass, J.J., Madimenos, F.C. \& Sugiyama, L.S. (2010). Life history, immune function, and intestinal helminths: trade-offs among immunoglobulin e, c-reactive protein, and growth in an amazonian population. Am.J. Hum. Biol., 22, 836-848.

Borer, E.T., Mitchell, C.E., Power, A.G. \& Seabloom, E.W. (2009). Consumers indirectly increase infection risk in grassland food webs. Proc. Natl Acad. Sci. USA, 106, 503-506.

Burnham, K.P. \& Anderson, D.R. (2002). Model Selection and Multimodel Inference. Springer-Verlag, New York, NY.

Craft, M.E., Hawthorne, P.L., Packer, C. \& Dobson, A.P. (2008). Dynamics of a multihost pathogen in a carnivore community. J. Anim. Ecol., 77, 12571264.

Cronin, J.P., Welsh, M.E., Dekkers, M.G., Abercrombie, S.T. \& Mitchell, C.E. (2010). Host physiological phenotype explains pathogen reservoir potential. Ecol. Lett., 13, 1221-1232.

Daszak, P., Cunningham, A.A. \& Hyatt, A.D. (2000). Wildlife ecology - emerging infectious diseases of wildlife: threats to biodiversity and human health. Science, 287, 443-449.

Frost, D.R., Grant, T., Faivovich, J., Bain, R.H., Haas, A., Haddad, C.F.B. et al. (2006). The amphibian tree of life. Bull. Am. Mus. Nat. Hist., 297, 8-370.

Gilbert, G.S. \& Webb, C.O. (2007). Phylogenetic signal in plant pathogen-host range. Proc. Natl Acad. Sci. USA, 104, 4979-4983.

Hart, B.L. (1994). Behavioral defense against parasites - interaction with parasite invasiveness. Parasitology, 109, S139-S151.

Hasselquist, D. (2007). Comparative immunoecology in birds: hypotheses and tests. J. Ornithol., 148, S571-S582. 
Hawley, D.M. \& Altizer, S.M. (2010). Disease ecology meets ecological immunology: understanding the links between organismal immunity and infection dynamics in natural populations. Funct. Ecol., 25, 48-60.

Haydon, D.T., Cleaveland, S., Taylor, L.H. \& Laurenson, M.K. (2002). Identifying reservoirs of infection: a conceptual and practical challenge. Emerg. Infect. Dis., 8, 1468-1473.

Johnson, P.T.J. \& Hartson, R.B. (2009). All hosts are not equal: explaining differential patterns of malformations in an amphibian community. J. Anim. Ecol., 78, 191-201.

Johnson, P.T.J., Lunde, K.B., Ritchie, E.G. \& Launer, A.E. (1999). The effect of trematode infection on amphibian limb development and survivorship. Science, 284, 802-804.

Johnson, P.T.J., Lunde, K.B., Ritchie, E.G., Reaser, J.K. \& Launer, A.E. (2001). Morphological abnormality patterns in a California amphibian community. Herpetologica, 57, 336-352.

Johnson, P.T.J., Kellermanns, E. \& Bowerman, J. (2011). Critical windows of disease risk: amphibian pathology driven by developmental changes in host resistance and tolerance. Funct. Ecol., 25, 726-734.

Jones, K.E., Patel, N.G., Levy, M.A., Storeygard, A., Balk, D., Gittleman, J.L. et al. (2008). Global trends in emerging infectious diseases. Nature, 451, 990-994.

Keesing, F., Holt, R.D. \& Ostfeld, R.S. (2006). Effects of species diversity on disease risk. Ecol. Lett., 9, 485-498.

Kilpatrick, A.M., Daszak, P., Jones, M.J., Marra, P.P. \& Kramer, L.D. (2006). Host heterogeneity dominates West Nile virus transmission. Proc. R. Soc. Lond. [Biol.], 273, 2327-2333.

Knowles, S.C.L., Nakagawa, S. \& Sheldon, B.C. (2009). Elevated reproductive effort increases blood parasitaemia and decreases immune function in birds: a meta-regression approach. Funct. Ecol., 23, 405-415.

Komar, N., Langevin, S., Hinten, S., Nemeth, N., Edwards, E., Hettler, D. et al. (2003). Experimental infection of North American birds with the New York 1999 strain of West Nile virus. Emerg. Infect. Dis., 9, 311-322.

LaDeau, S.L., Kilpatrick, A.M. \& Marra, P.P. (2007). West Nile virus emergence and large-scale declines of North American bird populations. Nature, 447, 710-714.

Lee, K.A. (2006). Linking immune defences and life history at the levels of the individual and the species. Integr. Comp. Biol., 46, 1000-1015.

Lee, K.A., Wikelski, M., Robinson, W.D., Robinson, T.R. \& Klasing, K.C. (2008). Constitutive immune defences correlate with life-history variables in tropical birds. J. Anim. Ecol., 77, 356-363.

Lochmiller, R.L. \& Deerenberg, C. (2000). Trade-offs in evolutionary immunology: just what is the cost of immunity? Oikos, 88, 87-98.

LoGiudice, K., Ostfeld, R.S., Schmidt, K.A. \& Keesing, F. (2003). The ecology of infectious disease: effects of host diversity and community composition on Lyme disease risk. Proc. Natl Acad. Sci. USA, 100, 567-571.

Martin, L.B., Hasselquist, D. \& Wikelski, M. (2006a). Investment in immune defense is linked to pace of life in house sparrows. Oecologia, 147, 565-575.

Martin, L.B., Weil, Z.M. \& Nelson, R.J. (2006b). Refining approaches and diversifying directions in ecoimmunology. Integr. Comp. Biol., 46, 1030-1039.

Martin, L.B., Weil, Z.M. \& Nelson, R.J. (2007). Immune defense and reproductive pace of life in Peromyscus mice. Ecology, 88, 2516-2528.

Martins, E.P. \& Hansen, T.F. (1997). Phylogenies and the comparative method: a general approach to incorporating phylogenetic information into the analysis of interspecific data. Am. Nat., 149, 646-667.

McGarigal, K., Cushman, S. \& Stafford, S.G. (2000). Multivariate Statistics for Wildlife and Ecology Research. Springer, New York.

Miller, M.R., White, A. \& Boots, M. (2007). Host life span and the evolution of resistance characteristics. Evolution, 61, 2-14.
Ostfeld, R. \& Keesing, F. (2000). The function of biodiversity in the ecology of vector-borne zoonotic diseases. Can. J. Zool., 78, 2061-2078.

Paradis, E., Bolker, B., Claude, J., Cuong, H.S., Desper, R., Durand, B. et al. (2011). Package 'ape', version 2.8. Analyses of phylogenetics and evolution. http:// ape.mpl.ird.fr/.

Poulin, R. \& Morand, S. (2004). Parasite Biodiversity. Smithsonian Books, Washington D.C.

Promislow, D.E.L. \& Harvey, P.H. (1990). Living fast and dying young - a comparative analysis of life history variation among mammals. J. Zool., 220, 417-437.

Purvis, A., Gittleman, J.L., Cowlishaw, G. \& Mace, G.M. (2000). Predicting extinction risk in declining species. Proc. R. Soc. Lond. [Biol.], 267, 1947-1952.

R Development Core Team (2008). R: A Language and Environment for Statistical Computing. R Foundation for Statistical Computing, Vienna, Austria. ISBN 3900051-07-0, URL http://www.R-project.org.

Råberg, L., Graham, A.L. \& Read, A.F. (2009). Decomposing health: tolerance and resistance to parasites in animals. Philos. Trans. R. Soc. Lond. [Biol.], 364, 37-49.

Ricklefs, R.E. \& Wikelski, M. (2002). The physiology/life-history nexus. Trends Ecol. Evol., 17, 462-468.

Rohr, J.R., Raffel, T.R. \& Hall, C.A. (2010). Developmental variation in resistance and tolerance in a multi-host-parasite system. Funct. Ecol., 24, 1110-1121.

Rosseel, Y., Oberski, D. \& Byrnes, J. (2011). Latent variable analysis v. 0.4-10. http://lavaan.org.

Schmid-Hempel, P. \& Ebert, D. (2003). On the evolutionary ecology of specific immune defence. Trends Ecol. Evol., 18, 27-32.

Sparkman, A.M. \& Palacios, M.G. (2009). A test of life-history theories of immune defence in two ecotypes of the garter snake, Thamnophis elegans. J. Anim. Ecol., 78, 1242-1248.

Stearns, S.C. (1992). The Evolution of Life Histories. Oxford University Press, New York.

Tieleman, B.I., Williams, J.B., Ricklefs, R.E. \& Klasing, K.C. (2005). Constitutive innate immunity is a component of the pace-of-life syndrome in tropical birds. Proc. R. Soc. Lond. [Biol.], 272, 1715-1720.

Todd, B.D. (2007). Parasites lost? An overlooked hypothesis for the evolution of alternative reproductive strategies in amphibians. Am. Nat., 170, 793-799.

Viney, M.E., Riley, E.M. \& Buchanan, K.L. (2005). Optimal immune responses: immunocompetence revisited. Trends Ecol. Evol., 20, 665-669.

Zuk, M. \& Stoehr, A.M. (2002). Immune defense and host life history. Am. Nat., 160, S9-S22.

\section{SUPPORTING INFORMATION}

Additional Supporting Information may be downloaded via the online version of this article at Wiley Online Library (www.ecologyletters.com).

As a service to our authors and readers, this journal provides supporting information supplied by the authors. Such materials are peer-reviewed and may be re-organised for online delivery, but are not copy-edited or typeset. Technical support issues arising from supporting information (other than missing files) should be addressed to the authors.

Editor, Peter Thrall

Manuscript received 27 October 2011

First decision made 5 December 2011

Manuscript accepted 12 December 2011 